\title{
TRABECULOTOMÍA-TRABECULECTOMÍA COMBINADA COMO TRATAMIENTO QUIRÚRGICO INICIAL DEL GLAUCOMA CONGÉNITO PRIMARIO
}

\section{COMBINED TRABECULOTOMY-TRABECULECTOMY AS THE INITIAL SURGICAL PROCEDURE OF PRIMARY DEVELOPMENTAL GLAUCOMA}

\author{
CAMPOS-MOLLO E ${ }^{1}$, MORAL-CAZALLA R ${ }^{1}$, BELMONTE-MARTÍNEZ J²
}

\section{RESUMEN}

Objetivo: Valorar los resultados quirúrgicos a largo plazo de la trabeculotomía-trabeculectomía combinada (TTC) como tratamiento preferente del glaucoma congénito primario.

Métodos: Se seleccionaron 22 ojos de 14 pacientes consecutivos con glaucoma congénito primario en los que se empleó la TTC como procedimiento inicial entre 1981 y 2005 . Se introdujo en una base de datos la información relacionada con la historia familiar, edad de inicio del glaucoma, síntomas y signos, integridad corneal, presión intraocular (PIO), gonioscopia, cirugía, complicaciones postoperatorias, agudeza visual, defecto de refracción, microscopia endotelial, longitud axial, campos visuales y estado final de la papila. El resultado de la presión intraocular fue evaluado usando el análisis de supervivencia Kaplan-Meier.

Resultados: Las probabilidades acumuladas de éxito después de realizar una TTC como procedimiento inicial fueron del $95,5 \%$ a los 12 meses y del $78,2 \%$ a los 24 meses, manteniéndose esta proporción durante 15 años de seguimiento. Cuatro ojos

\begin{abstract}
Purpose: To evaluate the long-term outcome of trabeculotomy-trabeculectomy as the primary surgical treatment for primary congenital glaucoma.

Methods: Twenty-two eyes of 14 consecutive patients with primary congenital glaucoma who underwent combined trabeculotomy-trabeculectomy as the initial procedure between 1981 and 2005 were selected for review. Records of ocular family history, age at onset of glaucoma, symptoms and signs, corneal integrity, intraocular pressure (IOP), gonioscopy, surgery, postoperative complications, visual acuity, refractive error, endothelial microscopy, axial length, visual fields and final status of the optic nerve head were entered into a computer database. The outcome, in terms of IOP obtained, was evaluated using Kaplan-Meier survival analysis.

Results: Cumulative probabilities of success, after performing combined trabeculotomy-trabeculectomy as the initial operative procedure, were $95.5 \%$ after 12 months and $78.2 \%$ after 24 months, with this rate being maintained during 15 years of followup. Four eyes $(18.1 \%)$ required more than one ope-
\end{abstract}

\footnotetext{
Recibido: 9/7/07. Aceptado: 25/6/08.

Servicio de Oftalmología. Hospital General Universitario de Alicante. Alicante. España.

1 Licenciado en Medicina.

2 Doctor en Medicina.

Comunicación presentada parcialmente en el LXXXI Congreso de la S.E.O. (Zaragoza 2005).

Correspondencia:

E. Campos-Mollo

Cl. Bañeres, $5,4^{\circ} \mathrm{D}$

03203 Elche (Alicante)

España

E-mail: ezechiel@hotmail.com
} 
$(18,1 \%)$ requirieron más de un procedimiento debido a una PIO elevada persistente. Las complicaciones postoperatorias fueron raras. Al final del seguimiento, de 12 ojos de 8 pacientes cooperadores, la mejor agudeza visual corregida fue igual o mayor de 0,5 en el $75 \%$ (9 ojos).

Conclusiones: La TTC como cirugía primaria ofrece una alta eficacia a largo plazo en el control de la PIO sin tratamiento farmacológico, mostrando mínimas complicaciones quirúrgicas y una baja incidencia de reintervenciones.

Palabras clave: Trabeculotomía-trabeculectomía, glaucoma congénito primario, buftalmos, glaucoma pediátrico, glaucoma infantil, glaucoma. rative procedure because of persistent raised IOP. Postoperative complications were rare. At the time of the last follow-up, of 12 eyes from 8 co-operative patients, the final best spectacle-corrected visual acuity was 0.5 (20/40) or better, in $75 \%$ (9 eyes).

Conclusion: Combined trabeculotomy-trabeculectomy as the primary surgical procedure offers longterm high efficacy in the control of IOP without medical treatment, is rarely associated with surgical complications, and a low need for re-operations (Arch Soc Esp Oftalmol 2008; 83: 479-486).

Key words: Trabeculotomy-trabeculectomy, primary congenital glaucoma, primary developmental glaucoma, buphthalmos, pediatric glaucoma, childhood glaucoma, glaucoma.

\section{INTRODUCCIÓN}

El término glaucoma congénito primario (GCP) está reservado para aquellos casos en los que existe un defecto anatómico del ángulo camerular debido a anomalías aisladas del desarrollo que dificultan la salida del humor acuoso (1). Constituye la forma más común de glaucoma infantil y exige un diagnóstico precoz y un tratamiento quirúrgico relativamente urgente al poder ocasionar graves secuelas morfológicas y funcionales.

Aunque, clásicamente, la goniotomía ha constituido la operación de primera elección, se han propuesto otras alternativas quirúrgicas como la trabeculotomía ab externo, la trabeculotomía-trabeculectomía o la trabeculectomía convencional.

La trabeculotomía-trabeculectomía combinada (TTC), también denominada trabeculo-trabeculectomía fue descrita para el GCP en 1979 por Nicolás Belmonte (2). Posteriormente varios autores han publicado resultados satisfactorios con este procedimiento (3-8).

La TTC es difícil de evaluar en las sociedades occidentales debido a su infrecuente uso y a la baja incidencia de GCP (1:10.000 recién nacidos). De hecho, la efectividad de esta técnica se ha analizado en escasas publicaciones y la mayoría de los estudios se han realizado en poblaciones árabes donde la incidencia de GCP es mayor a causa de la alta consanguinidad (1:2.500 recién nacidos) (9).

Durante los últimos 25 años, hemos empleado la TTC como tratamiento primario de elección con aparente buen resultado inicial por lo que nos pro- pusimos comprobar retrospectivamente su eficacia a largo plazo con un seguimiento medio de 8,9 años (siendo inferior a 5 años en el resto de las series publicadas).

\section{SUJETOS, MATERIAL Y MÉTODOS}

Se revisaron un total de 22 ojos (designados de 1 a 22) de 14 pacientes consecutivos (designados de I a XIV) diagnosticados e intervenidos de GCP en el Hospital General Universitario de Alicante desde enero de 1981 hasta diciembre de 2005. Se evaluó toda la información preoperatoria y postoperatoria, así como los resultados quirúrgicos a largo plazo. Todas las intervenciones fueron realizadas por el mismo cirujano (JBM).

Como el número de otros procedimientos fue insuficiente para permitir un análisis estadístico comparativo entre las diferentes técnicas, los pacientes que se sometieron a otra cirugía como tratamiento inicial fueron excluidos. También se excluyeron aquellos pacientes con glaucomas pediátricos asociados a otras anomalías oculares o enfermedades sistémicas.

Se creó una base de datos que incluyó la historia familiar, edad de inicio del glaucoma, síntomas y signos, integridad corneal, presión intraocular (PIO), gonioscopia, cirugía, complicaciones postoperatorias, agudeza visual, defecto de refracción, microscopia endotelial, longitud axial, campos visuales, estado final de la papila, seguimiento y resultado final. 
El diagnóstico preoperatorio se basó en los síntomas y signos clínicos precoces (fotofobia, blefaroespasmo, lagrimeo, edema corneal y aumento del tamaño corneal). La presión intraocular se midió con el tonómetro de aplanación de Perkins bajo anestesia con halotano en cuanto el niño se encontró suficientemente anestesiado para permitir su medición (durante los 10 primeros minutos después de la inducción y antes de la intubación traqueal).

La medición de la presión intraocular (PIO) bajo anestesia se realizó a los 6 meses de la cirugía y posteriormente de forma anual. Los niños mayores y más cooperadores fueron explorados usando el tonómetro de Goldmann.

El criterio diagnóstico de fracaso quirúrgico se estableció cuando se necesitó una cirugía adicional debido a una presión intraocular por encima de $21 \mathrm{mmHg}$ (incluso con medicación tópica) o una PIO superior a $16 \mathrm{mmHg}$ bajo anestesia general con persistencia de otros condicionantes como síntomas (fotofobia, lagrimeo) y signos (buftalmos, progresión de la excavación o atrofia del nervio óptico). Las probabilidades acumulativas de éxito se determinaron mediante el análisis de supervivencia de Kaplan-Meier (SPSS v.14, SPSS Inc., Chicago, Illinois, USA).

Debido a la corta edad de algunos pacientes no pudimos efectuar algunas pruebas complementarias. Cuando fue posible, en base a la edad y colaboración del paciente se valoró el defecto de refracción, biomicroscopia, estado del nervio óptico, la microscopía endotelial (SP 2000, Topcon, Tokio, Japón), la longitud axial (Ocuscan, Alcon, Irvine, Cal, USA) y el campo visual (Humphrey, Zeiss, Dublín, CA). Un total de 8 pacientes (12 ojos) cooperaron suficientemente para obtener exploraciones fiables.

\section{Técnica quirúrgica}

La intervención se inicia, bajo anestesia general, dando un punto de recto superior con seda de 4/0 para facilitar la exposición de la conjuntiva superior. A continuación se crea un colgajo conjuntival con base en fórnix posicionado en el limbo superior (a la I o las XI). Después, se diseca un tapete rectangular hacia el limbo de un tamaño aproximado de 2 x $3 \mathrm{~mm}$ profundizando hasta la mitad o dos tercios del espesor escleral.

Usando una alta magnificación, en la esclera subyacente, se realiza cuidadosamente una incisión radial rasgando con la porción roma de un cuchille- te (fig. 1). La incisión se efectúa en la zona de transición entre la córnea azul (banda trabecular) y el tejido escleral donde teóricamente el canal de Schlemm se encuentra localizado. El canal es identificado al observar un reflujo de humor acuoso desde la incisión, ocasionalmente mezclado con sangre.

En ocasiones puede ser difícil detectar el canal de Schlemm debido a un anormal alargamiento anatómico del limbo y a una esclera excesivamente adelgazada en ojos buftálmicos.

Las tijeras de Vannas son introducidas a nivel de la incisión del canal expuesto, seccionándolo $1 \mathrm{~mm}$ a cada lado. El trabeculotomo doble, derecho e izquierdo (Sourdille-Paufique, Moria ${ }^{\circledR}$ ) es insertado en la incisión comprobándose su avance en el lumen del canal sin resistencia. A continuación, se rota de forma controlada hacia la cámara anterior, atravesando, de esta forma, la cara interna del canal de Schlemm, rompiendo la malla trabecular y el tejido embrionario del ángulo (fig. 2), debiendo evitar lesionar otras estructuras intraoculares. De manera similar se introduce el trabeculotomo por el otro extremo de la incisión (fig. 3). La pared interna del canal de Schlemm es seccionada finalmente en una extensión de unos $120^{\circ}$.

Posteriormente en el lecho córneo-escleral se escinde una pequeña pieza de tejido de aproximadamente 1 x $2 \mathrm{~mm}$ incluyendo malla trabecular y una porción del canal de Schlemm (trabeculectomía), seguida de una iridectomía periférica (fig. 4).

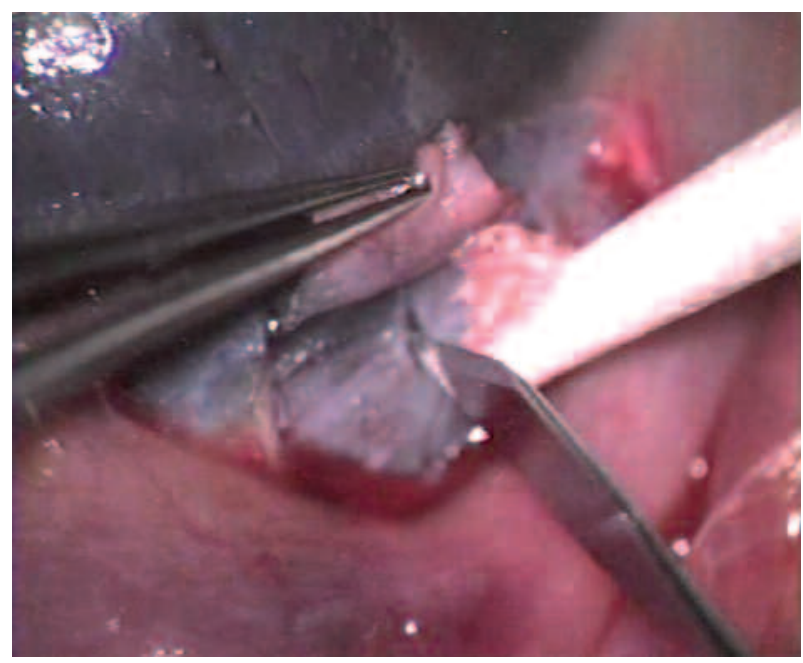

Fig. 1: Se realiza cuidadosamente una incisión radial rasgando con un cuchillete en la zona de transición entre la córnea azul (banda trabecular) y el tejido escleral para identificar el canal de Schlemm. 


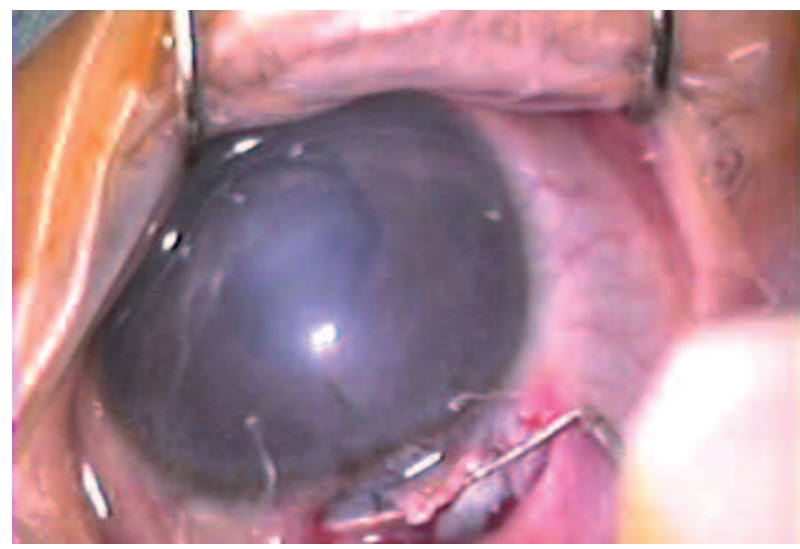

Fig. 2: El trabeculotomo se introduce en el canal de Schlemm y se rota de forma controlada hacia la cámara anterior rompiendo la malla trabecular.

El tapete escleral es reposicionado suturándolo con dos puntos interrumpidos en sus esquinas de nylon de 10/0 y enterrando los nudos. La conjuntiva es cerrada con suturas reabsorbibles interrumpidas o continuas de 8/0.

Después de la cirugía se aplican gotas de antibiótico y corticoide. El ojo se ocluye en casos unilaterales, omitiéndose en los bilaterales. El tratamiento postoperatorio se continúa durante tres o cuatro semanas, disminuyendo las pautas de acuerdo con el aspecto clínico y la evolución.

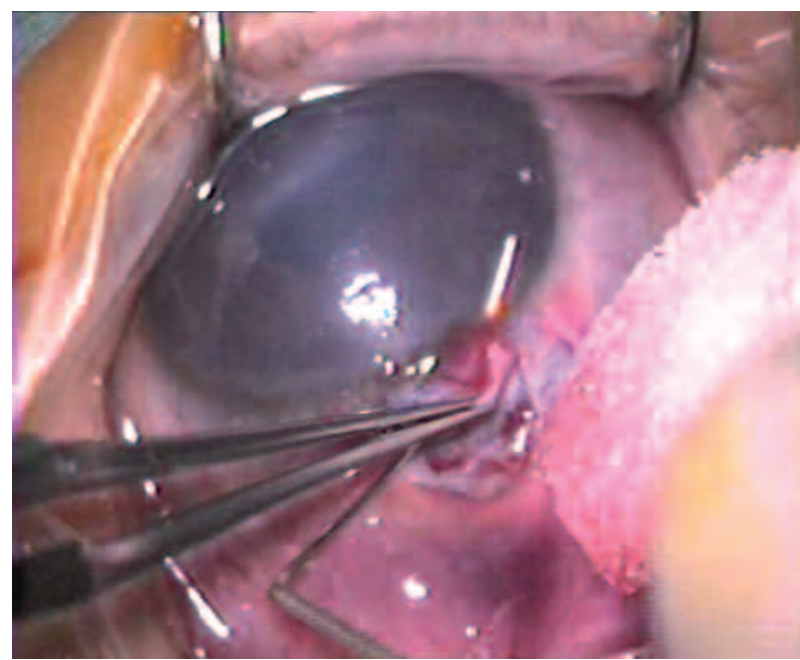

Fig. 3: A continuación, el trabeculotomo se introduce y se rota por el otro extremo de la incisión.

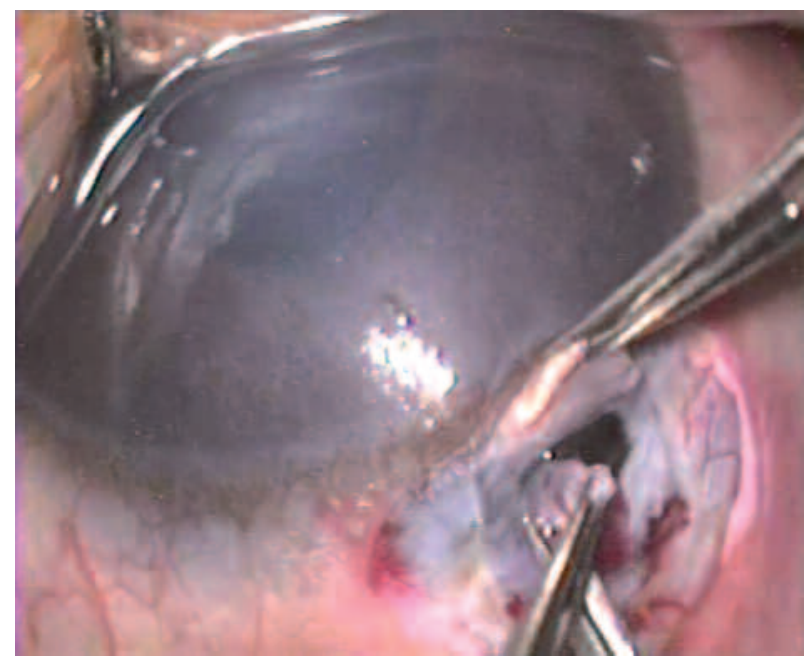

Fig. 4: Posteriormente, se efectúa la trabeculectomía seguida de una iridectomía periférica.

\section{RESULTADOS}

Se analizaron un total de 22 ojos de 14 pacientes, 10 varones $(71,4 \%)$ y 4 mujeres $(28,5 \%)$. La enfermedad fue bilateral en 8 niños $(57,1 \%)$ y unilateral en $6(42,8 \%)$. Sólo un paciente presentó antecedentes familiares de GCP. De los casos unilaterales el OD se afectó en un $66,6 \%$ y el OI en un $33,3 \%$.

La edad en el momento del diagnóstico varió desde 1 día hasta 23,5 meses (mediana 2,3 meses). La sospecha de glaucoma congénito se basó en el aumento del tamaño corneal (22 ojos; 100\%), edema corneal (14 ojos; 63,6\%), lagrimeo (12 ojos; $54,5 \%$ ), fotofobia y blefaroespasmo (13 ojos; $59 \%$ ). El diámetro corneal horizontal medio fue de 13,4 $\mathrm{mm} \pm 1,1$ (rango de 12 a $15 \mathrm{~mm}$ ). La PIO media preoperatoria antes de la primera cirugía fue de 20,1 $\pm 4,2 \mathrm{~mm} \mathrm{Hg}$. El tiempo de seguimiento se extendió desde 1 año a 24 años con una media de 8,9 años (118,8 meses). En uno de los niños se objetivó un nistagmo de fijación.

La probabilidad acumulativa de éxito para la PIO con un único procedimiento fue de un $95,5 \%$ a los 12 meses y un 78,2\% a los 24 manteniéndose esta proporción durante 15 años de seguimiento. Si bien, sólo 5 pacientes (8 ojos) completaron los 10 años de seguimiento. La mayoría de los fracasos quirúrgicos ocurrieron en los dos primeros años (fig. 5).

Cuatro ojos $(18,1 \%)$ requirieron más de una intervención debido a una PIO elevada a pesar del tratamiento tópico hipotensor. La técnica quirúrgica 


\section{Función de supervivencia Kaplan-Meier}

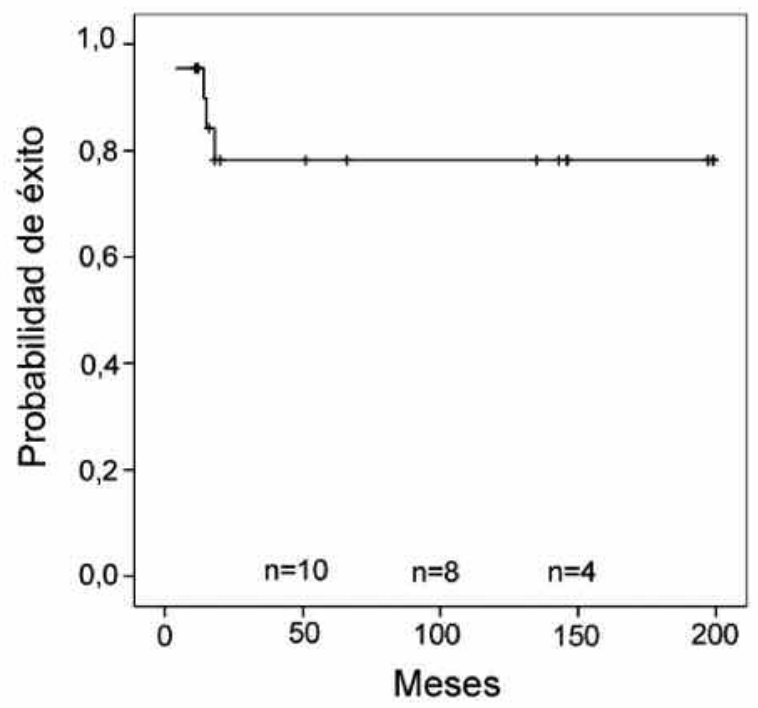

Fig. 5: La curva de supervivencia Kaplan-Meier muestra la estimación de éxito en el control de la PIO después del primer procedimiento para el glaucoma congénito primario. El número de ojos (n) que permanecen con riesgo de fracaso a distintos intervalos están indicados a lo largo del eje horizontal.

de elección para todas las reoperaciones fue la trabeculectomía. Después de una segunda operación la PIO se normalizó en 2 ojos, precisando uno de ellos un betabloqueante tópico adicional. En los 2 ojos restantes, de un mismo paciente (paciente I), no se controló la tensión ocular. Una tercera intervención fue realizada en otro centro hospitalario evolucionando uno de ellos a una ptisis bulbi y padeciendo el otro un trauma severo con hemoftalmos y desprendimiento de retina que obligó a su evisceración.

Durante la cirugía, al abrir el canal de Schlemm, usualmente se observó un sangrado insignificante en cámara anterior. Sólo en 2 ojos se produjo un hifema moderado que se resolvió en pocos días sin consecuencias.

En 3 ojos se produjo un atrapamiento del iris a la incisión. En un ojo, se asoció a ectropión uveal sin consecuencias. Otro ojo precisó una sinequiotomía y otro, con una marcada corectopia, requirió sinequiolisis y coreoplastia. Después de diez años, este último, desarrolló una catarata subcapsular anterior periférica, que no requirió cirugía debido a la mínima repercusión sobre la visión.
En un ojo, cuatro años después del procedimiento, se detectó una subluxación de cristalino precisando extracción extracapsular e implante de LIO de cámara posterior. Posteriormente, se opacificó la cápsula posterior requiriendo una capsulotomía con láser YAG. Después desarrolló una vitreorretinopatía proliferativa siendo intervenido con mal resultado funcional. Este mismo ojo, sufrió luego un traumatismo accidental con laceración corneal e hifema que se resolvió espontáneamente en pocos días, manteniéndose la tensión ocular controlada pero con pérdida total de la visión.

Solamente 3 de los 22 ojos (10,9\%) tuvieron complicaciones graves con una evolución final desfavorable, dos de ellos correspondientes a un mismo paciente.

La PIO media en la última medición bajo anestesia general fue $10,89 \pm 4,1 \mathrm{mmHg}$ implicando una descenso medio de $10,82 \mathrm{mmHg} \pm 4,36 \mathrm{mmHg}$. Al final del seguimiento, se consiguió un control de la PIO en 20 ojos $(90,9 \%)$ después de una o dos intervenciones y únicamente uno requirió un hipotensor tópico adicional. En los ojos con PIO controlada, todas las ampollas, fueron difusas o ligeramente elevadas.

No se percibieron variaciones en el diámetro horizontal de las córneas (media 13,4 \pm 1,3 mm) en comparación con las mediciones preoperatorias. $\mathrm{Al}$ final del seguimiento, 13 ojos $(59,05 \%)$ mostraron una córnea transparente; 5 ojos $(22,7 \%)$ presentaron una opacidad corneal leve, con transparencia aceptable; y 2 ojos $(9,09 \%)$ mostraron una opacidad difusa persistente. Por razones obvias no se pudieron evaluar las dos córneas $(9,09 \%)$ del paciente I comentado anteriormente.

Doce ojos de 8 pacientes colaboradores presentaron criterios para estudiar la agudeza visual, biomicroscopia, PIO medida con tonómetro de Goldmann, microscopia endotelial, longitud axial y campimetria. El paciente I a pesar de una buena cooperación, fue excluido del análisis. El resumen de los datos exploratorios se muestra en la tabla I.

La mejor agudeza visual con corrección fue igual o superior a 0,5 en 9 ojos $(75,0 \%)$ e inferior a 0,1 en 2 ojos (16,6\%). La pérdida de visión se atribuyó principalmente a las opacidades corneales y a la ambliopía. La oftalmoscopia mostró un aumento de la excavación papilar en 2 ojos. Sólo se pudieron obtener datos campimétricos fiables en 4 ojos, no apreciándose alteraciones campimétricas significativas. 
Tabla I. Resultados a largo plazo en pacientes con GCP que cooperaron suficientemente para la realización de exploraciones fiables

\begin{tabular}{|c|c|c|c|c|c|c|c|c|}
\hline $\mathrm{O} / \mathrm{P}$ & Edad (años) & $\mathrm{AVc}$ & $\mathrm{PIO} \neq$ & Córnea & EP & DEC & LA & $\mathrm{CV}$ \\
\hline 3/II & 17 & 1 & 13 & $\mathrm{~T}$ & $2 / 10$ & 3228 & 24,02 & $\mathrm{~N}$ \\
\hline 4/II & 17 & 1 & 12 & $\mathrm{~T}$ & $2 / 10$ & 2694 & 24,08 & $\mathrm{~N}$ \\
\hline 5/III & 16 & 1 & 14 & OCL & $3 / 10$ & 1784 & 23,68 & $\mathrm{~N}$ \\
\hline 6/III & 16 & 0,7 & 17 & OCL & $4 / 10$ & 1509 & 25,17 & $\mathrm{~N}$ \\
\hline 7/IV & 12 & 0,8 & 17 & OCL & $3 / 10$ & 1645 & 26,23 & NV \\
\hline 8/IV & 12 & 0,8 & 18 & OCL & $7 / 10$ & 1940 & 27,14 & NV \\
\hline $9 / \mathrm{V}$ & 12 & $0,3 *$ & $15 \S$ & $\mathrm{T}$ & NR & 1421 & 23,92 & NV \\
\hline $10 / \mathrm{V}$ & 12 & PL * & 14 & OCE & NR & NR & 24,12 & NR \\
\hline 11/VI & 11 & 0,5 & 10 & $\mathrm{~T}$ & $2 / 10$ & 3187 & 23 & NV \\
\hline $12 / \mathrm{VII}$ & 6 & 0,9 & 14 & $\mathrm{~T}$ & $2 / 10$ & 2476 & 24,85 & NR \\
\hline 13/VIII & 5 & $0,05 \dagger$ & 20 & OCL & $3 / 10$ & NR & 24,61 & NR \\
\hline 14/IX & 5 & 0,5 & 16 & $\mathrm{~T}$ & $2 / 10$ & NR & NR & NR \\
\hline
\end{tabular}

$\mathrm{O} / \mathrm{P}=$ ojo/paciente; $\mathrm{AVc}=$ agudeza visual corregida; $\mathrm{PL}=$ percepción luminosa; $\mathrm{EP}=$ excavación papilar; $\mathrm{DEC}=$ densidad endotelial corneal (células $\left./ \mathrm{mm}^{2}\right) ; \mathrm{LA}=$ longitud axial $(\mathrm{mm}) ; \mathrm{CV}=$ campo visual; $\mathrm{T}=$ transparente; $\mathrm{OCL}=$ opacidad corneal leve; $\mathrm{OCE}=$ opacidad corneal estromal $\mathrm{NR}=$ no realizable; $\mathrm{NV}=$ no valorable; $\mathrm{N}=$ normal. *Ojo con intensa fotofobia a pesar de una PIO controlada. $\dagger$ Ambliopía debido a la falta de colaboración por parte de la familia. \$ PIO postoperatoria medida con tonómetro de Goldmann $(\mathrm{mm} \mathrm{Hg})$. § PIO controlada con medicación tópica.

\section{DICUSIÓN}

La cirugía es el único tratamiento eficaz para el GCP siendo la goniotomía ab interno la técnica preferida por muchos cirujanos cuando la córnea es lo suficiente transparente como para permitir una adecuada visualización del ángulo camerular (10-13). Por otro lado, en la goniotomía el canal de Schlemm no es seccionado, no actuándose sobre determinadas alteraciones histopatológicas descritas en el GCP como la inmadurez de la malla trabecular o del canal de Schlemm $(1,14,15)$. Estos eventos podrían explicar, en algunos casos, el fracaso de una primera intervención y la necesidad de repetir el procedimiento.

La TTC crea dos vías de salida del acuoso: la primera (trabeculotomía) secciona el espesor total de la malla trabecular proporcionando una comunicación entre la cámara anterior y el canal de Schlemm; y la segunda (trabeculectomía) permite crear una fístula suplementaria permitiendo el drenaje desde la cámara anterior al espacio subconjuntival $(5,7)$. Esta doble vía de salida justificaría, al menos parcialmente, la alta incidencia de éxito en el control de la PIO con un único procedimiento $(4,5)$.

La TTC tiene además una significativa ventaja en aquellos casos en los que la visualización del ángulo está limitada por la opacidad corneal $(63,6 \%$ en nuestra serie), actuándose sobre una zona que el cirujano de segmento anterior domina bien bajo el microscopio.

Sus inconvenientes, sin embargo, son la dificultad técnica de disecar un colgajo escleral lamelar en un ojo muy adelgazado por el buftalmos, la localización e identificación precisa del canal de Schlemm para introducir el trabeculotomo en casos de megalocórnea extrema, con marcada alteración del área límbica y la ruptura ab externo «a ciegas» del canal de Schlemm y del tejido trabecular al carecer de la visualización directa del ángulo a diferencia de la goniotomía. De todos modos, aún cuando la identificación del canal de Schlemm o su canalización con el trabeculotomo no se haya realizado de forma precisa, la trabeculectomía suplementaria proporciona una vía adicional para la salida del humor acuoso que disminuiría la PIO a través de un mecanismo de filtración. Finalmente, al no respetarse la conjuntiva perilímbica se limitan las áreas para una futura intervención en el caso de un fracaso quirúrgico.

El control microscópico minucioso, la mayor familiaridad con el área quirúrgica, los buenos resultados desde su inicio con un único procedimiento y la baja incidencia de complicaciones, nos indujo a escoger la TTC como procedimiento primario de elección tanto en las córneas opacas como en las transparentes.

El análisis favorable de nuestra serie se apoya fundamentalmente en los excelentes resultados quirúrgicos a largo plazo (media 8,9 años), la baja tasa de reintervenciones, el buen control tensional, la remisión de los signos y síntomas y la recuperación funcional visual, similares a otros estudios en los que incluso el período de seguimiento fue mucho más corto (3-8). 
En la práctica, las complicaciones de la TTC no son mayores que las registradas con cada método por separado (7). Un hifema leve fue especialmente prevalente, no observándose atalamias o problemas relacionados con la ampolla de filtración (infección, ampollas adelgazadas, perforación, etc.). El enclavamiento del iris a nivel de la trabeculectomía sólo fue significativo en un ojo.

La mayoría de los fracasos quirúrgicos ocurrieron en los dos primeros años planificándose una nueva trabeculectomía estándar como segundo procedimiento. La naturaleza subyacente de la enfermedad y el diagnóstico precoz influyen ampliamente en la evolución y pronóstico de cada caso (16). En los tres ojos (dos del mismo paciente) que tuvieron un resultado final desfavorable posiblemente intervino la naturaleza de la malformación y la mayor dilación en el tratamiento quirúrgico primario. Por otro lado, es evidente que, en estos ojos, una presión intraocular controlada, no asegura un buen resultado funcional por lo que es preciso prestar atención individualizada a cada paciente tratando la anisometropía o la posible ambliopía mediante una corrección óptica precoz y, eventualmente, ejercicios de oclusión.

El diseño retrospectivo y el reducido número de pacientes constituye una de las limitaciones de este estudio que impide, con la información disponible, obtener conclusiones definitivas. No obstante, en nuestra opinión, la TTC es una técnica suficientemente segura, efectiva y predecible para poder adoptarse como primera opción en el tratamiento quirúrgico del GCP, al menos si no se domina o resulta problemática la goniotomía convencional.

\section{BIBLIOGRAFÍA}

1. Maul E, Strozzi L, Muñoz C, Reyes C. The outflow pathway in congenital glaucoma. Am J Ophthalmol 1980; 89: 667-673.
2. Belmonte N, Ladislao-Pérez J. Trabeculo-trabeculectomía. Arch Soc Esp Oftalmol 1979; 39: 610-614.

3. Turut P, Ribstein G, Milazzo S, Madelain J. Combined trabeculotomy and trabeculectomy in primary congenital glaucoma. Bull Soc Ophtalmol Fr 1988; 88: 1021-1024.

4. Elder MJ. Combined trabeculotomy-trabeculectomy compared with primary trabeculectomy for congenital glaucoma. Br J Ophthalmol 1994; 78: 745-748.

5. Mandal AK, Naduvilath TJ, Jayagandan A. Surgical results of combined trabeculotomy-trabeculectomy for developmental glaucoma. Ophthalmology 1998; 105: 974982.

6. Biedner BZ, Rothkoff L. Combined trabeculotomy-trabeculectomy compared with primary trabeculotomy for congenital glaucoma. J Pediatr Ophthalmol Strabismus 1998; 35: 49-50.

7. Mullaney PB, Selleck C, Al-Awad A, Al-Mesfer S, Zwaan $J$. Combined trabeculotomy and trabeculectomy as an initial procedure in uncomplicated congenital glaucoma. Arch Ophthalmol 1999; 117: 457-460.

8. Al-Hazmi A, Awad A, Zwaan J, Al-Merfer SA, Al-Jadaan I, Al-Mohamed A. Correlation between surgical success rate and severity of congenital glaucoma. $\mathrm{Br} J$ Ophthalmol 2005; 89: 449-453.

9. Ho CL, Walton DS. Primary congenital glaucoma: 2004 update. J Pediatr Ophthalmol Strabismus 2004; 41: 271 288.

10. Barkan O. Surgery of congenital glaucoma; review of 196 eyes operated by goniotomy. Am J Ophthalmol 1953; 36: 1523-1534.

11. Morgan KS, Black B, Ellis FD, Helveston EM. Treatment of congenital glaucoma. Am J Ophthalmol 1981; 92: 799803.

12. Broughton WL, Parks MM. An analysis of treatment of congenital glaucoma by goniotomy. Am J Ophthalmol 1981; 91: 566-572.

13. García-Sánchez J, Zato-Gómez de Liaño MA, FernándezVila PC. Glaucoma congénito. Arch Soc Esp Oftalmol 1984; 46: 245-250.

14. Tawara A, Inomata H. Developmental immaturity of the trabecular meshwork in congenital glaucoma. Am J Ophthalmol 1981; 92: 508-525.

15. Mumenee AE. The pathogenesis of congenital glaucoma; a new theory. Am J Ophthalmol 1959; 47: 827-858.

16. Dietlein TS, Jacobi PC, Krieglstein GK. Prognosis of primary ab externo surgery for primary congenital glaucoma. Br J Ophthalmol 1999; 83: 317-322. 